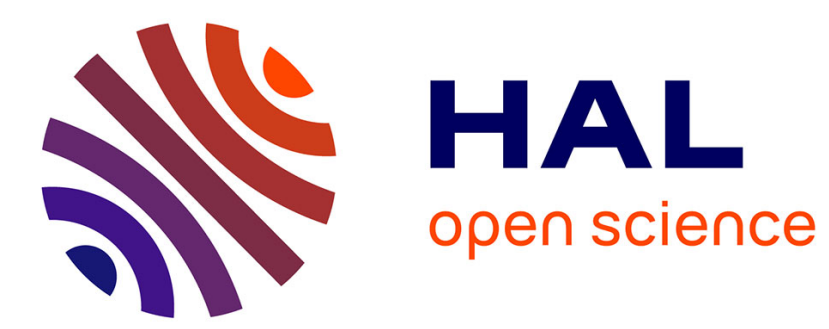

\title{
Automatic palpation for quantitative ultrasound elastography by visual servoing and force control
}

Pedro Patlan-Rosales, Alexandre Krupa

\section{To cite this version:}

Pedro Patlan-Rosales, Alexandre Krupa. Automatic palpation for quantitative ultrasound elastography by visual servoing and force control. IEEE/RSJ Int. Conf. on Intelligent Robots and Systems, IROS'16, Oct 2016, Daejeon, South Korea. pp.2357-2362. hal-01355406

\author{
HAL Id: hal-01355406 \\ https://hal.inria.fr/hal-01355406
}

Submitted on 23 Aug 2016

HAL is a multi-disciplinary open access archive for the deposit and dissemination of scientific research documents, whether they are published or not. The documents may come from teaching and research institutions in France or abroad, or from public or private research centers.
L'archive ouverte pluridisciplinaire HAL, est destinée au dépôt et à la diffusion de documents scientifiques de niveau recherche, publiés ou non, émanant des établissements d'enseignement et de recherche français ou étrangers, des laboratoires publics ou privés. 


\title{
Automatic palpation for quantitative ultrasound elastography by visual servoing and force control
}

\author{
Pedro A. Patlan-Rosales ${ }^{1}$ and Alexandre Krupa ${ }^{2}$
}

\begin{abstract}
The precise location of tumors is an important step in surgical planning that can be obtained from mechanical properties of soft tissues. In this paper we propose a roboticassisted palpation system that automatically moves an ultrasound probe to optimize the elastography process and improve the resulting elastogram. The main contribution of this work is the use of the elastography modality directly as input of the robot controller. Force measures are also considered in the probe control in order to automatically induce soft tissue deformation needed for real-time elastography imaging process. Moreover, an automatic exploration process is implemented to orient the probe to reach different views of a soft tissue target of interest. This allows to improve the elastogram quality of the element of interest by fusing the information observed from different positions.
\end{abstract}

\section{INTRODUCTION}

Ultrasonography is a popular medical imaging technique with many clinical applications. Its properties of portability, no ionising radiation, real-time acquisition, and low cost are very attractive in clinical practice. The ultrasound is well known for the propagation of mechanical waves on high frequency compression. It allows the construction of morphological images of organs, but lacks fundamental quantitative information of the elastic properties of tissue.

Ultrasound elastography is a non-invasive technique that estimates tissue stiffness and provides additional clinical information. For these reasons, elastography has gained interest on diagnosis of diseases, monitoring of ablation treatment for hepatocellular carcinoma [1], liver tumor ablation [2], estimation of mechanical properties of tissue [3], diagnosis of benign and malignant thyroid modules [4] and liver fibrosis [5]. Moreover, ultrasound elastography does not necessarily require special hardware or major alterations in the equipment, therefore, it can be integral part of any ultrasound system, from large cart-based to pocket size systems.

Since 2012, the concept of using robotic systems to facilitate the elastography process has been considered. A robotassisted system was proposed for real-time laparoscopic ultrasound elastography [6]. It showed an improvement with respect to the manual palpation for elastography using the da Vinci S surgical robot (Intuitive Surgical Inc., Sunnyvale, CA). Another system was proposed for laparoscopic ultrasound elastography using a vibrator positioned on the patient body instead of palpation [7].

\footnotetext{
${ }^{1}$ Pedro A. Patlan-Rosales is with Inria Rennes-Bretagne Atlantique, Universite de Rennes 1, campus universitaire de Beaulieu, Rennes 35042, France. pedro-alfonso.patlan-rosalesdinria.fr

${ }^{2}$ Alexandre Krupa is with Inria Rennes-Bretagne Atlantique, campus universitaire de Beaulieu, Rennes 35042, France. alexandre.krupa@inria.fr
}

Recently, the force control of a robot that allows performing a consistent pre-loading was considered in order to obtain an elasticity image [8]. Also, a robot-assisted system was proposed in [9] as a solution for segmenting rigid objects in elastographic images based on a machine learning algorithm. These previous works showed clearly that the use of a robotic system can improve the elastography process.

In this paper, we propose a control scheme that combines visual information and force measurement to automatically move an ultrasound probe mounted on a robotic arm to generate the palpation motion needed for quantitative elastography. The idea to use visual information extracted from medical image modalities in a robotic control scheme, specially in B-mode ultrasound, has already been presented in several works. Recently, a visual servoing model using ultrasound confidence maps was proposed in [10] to improve the ultrasound image quality with excellent performance.

The aim of our work is to automatize the palpation motion required to perform the elastography of a region of interest (ROI) in the observed ultrasound image. We design a hybrid force-vision control approach that considers the current estimated strain map as visual features. The robotic task we propose consists in automatically moving the US probe to reach different view angles of an element of interest while generating tissue compression required for the elastography process. Warping and averaging the obtained strain maps improves then the elastogram quality. Such improvement of the elastogram quality was already considered without robotic assistance in [11] where a spatialangular compounding elastography based on multiple US beam steering was introduced. To the best of our knowledge, it is the first time elastogram images are directly used in a robotic closed-loop control. In Section II-A, we first present an introduction to the elastography process and how we estimate the elastograms. Section II-B details our control approach based on force and elastogram information to automatically control 3 degrees of freedom (3 DOF in the image plane) of a robotized ultrasound probe. Experimental results validating our method are presented and discussed in Section III.

\section{METHODS}

\section{A. Ultrasound Elastography}

Mechanical properties of an organ or tissue are essential for the diagnosis in medicine. For example, a tumor or diseased tissue can be detected by its stiffness, generally perceive by palpation. However, this method is limited by the accessibility of the fingers to the tissue of interest, 
and it is only qualitative and distorted by surrounding tissues. Elastography is an approach able to overcome these challenges. The term elastography was coined by Ophir et al. [12], who proposed to use the echo waves generated by a piezoelectric transducer array (an ultrasound probe) after travelling through the tissues. The waves can be defined as radio-frequency signals ( $R F$ signal) and modeled as a succession of springs (see Fig. 1a). (a)

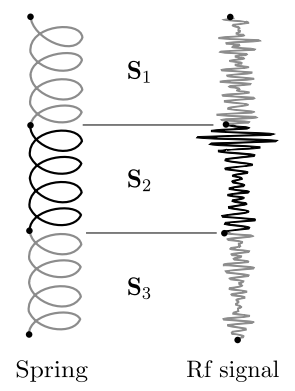

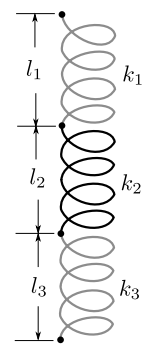

(b)

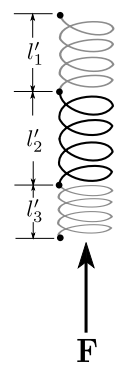

methods are used to compute an approximation of the motion field from time-varying signal amplitude. OF is defined as:

$$
\nabla I \cdot \mathbf{u}+I_{t}=0
$$

where $\nabla I$ denotes the spatial gradient of the signal amplitude, $I_{t}$ is its temporal variation and $\mathbf{u}$ corresponds to the motion parameters (in two dimensions).

Let $f(x, y)$ and $g(x, y)$ being the pre- and postcompressed signals in the ROI (see Fig. 2), we obtain then:

$$
\begin{aligned}
\nabla I(x, y) & =\left[\begin{array}{ll}
\frac{\partial f(x, y)}{\partial x} & \frac{\partial f(x, y)}{\partial y}
\end{array}\right], \\
I_{t} & =g(x, y)-f(x, y) \text { and } \\
\mathbf{u} & =\left[\begin{array}{ll}
u & v
\end{array}\right]^{T} .
\end{aligned}
$$

and the OF equation (2) becomes:

$$
\left[\begin{array}{ll}
\frac{\partial f(x, y)}{\partial x} & \frac{\partial f(x, y)}{\partial y}
\end{array}\right]\left[\begin{array}{ll}
u & v
\end{array}\right]^{T}=f(x, y)-g(x, y)
$$

Fig. 1. (a) RF signal analogy with a succession of springs $\mathbf{S}_{1}, \mathbf{S}_{2}$ and $\mathbf{S}_{3}$. (b) the Hooke's law scheme.

Elastography is performed in a rectangular ROI of an array of RF signals that are measured by an ultrasound probe and acquired by an ultrasound machine (see Fig. 2). Then, the ROI dimensions and its position are kept over two or more consecutive arrays of RF signals. If an axial force is applied over the succession of springs (see Fig. 1b), the length in each spring will change according to Hooke's law, as follows:

$$
F=\sum_{i} k_{i} \Delta l_{i}
$$

where $k_{i}$ is the spring constant for the $i$-th spring, and $\Delta l_{i}$ is the deformation of each spring. The strain value for each $i$-th spring is defined as $\varepsilon_{i}=\frac{\Delta l_{i}}{l_{i}}$. Fig. 1b shows the Hooke's law scheme, where $l_{i}$ is the initial length of the $i$-th spring, $l_{i}^{\prime}$ is the length after a stress is applied and $\Delta l_{i}=l_{i}^{\prime}-l_{i}$ is the difference of lengths.

In our case, the Hooke's law is adapted to the echo signals and the change of length $\Delta l$ is the time-delay $\Delta_{t}$ between the pre- and post-compression segment of RF signal. The improvement of the estimation of $\Delta_{t}$, also known as motion estimation, is the key to compute a good strain map.

Time delay estimation (TDE) is commonly based on the normalized cross-correlation (NCC) as proposed Ophir's work [12]. Additionally, there are other ways to estimate the TDE: phase zero estimation (PZE) [13], axial velocity estimation (AVE) [14] and optical flow (OF) [15].

The disadvantage of the existing solutions is the effect of the lateral motion on the motion estimation, which is computed in axial way. The search region can be tuned in the approaches to include the lateral displacement, but the computational cost will grow considerably. OF has advantages over the other methods. It can be applied directly to the RF signals array whereas AVE and PZE require a previous $\mathrm{RF}$ signal interpolation. OF was first proposed as a motion estimation technique in computer vision by Horn [16]. OF

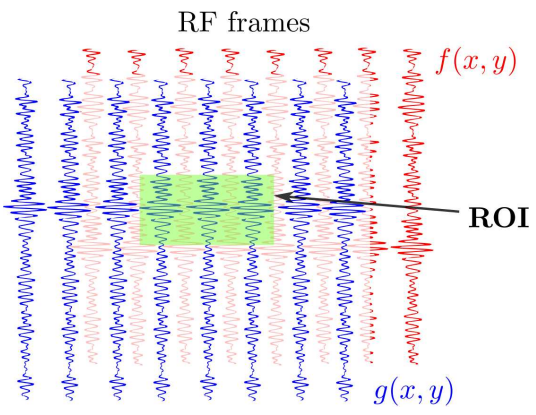

Fig. 2. RF frames within a region of interest.

The ROI is divided in a set of windows (width $w_{s} \geq 1$ and height $h_{s} \geq 1$ ) that are positioned with at least $25 \%$ of overlapping (Fig. 2). The values of the displacements $u$ and $v$ are estimated by solving (6) using least-squares for each window inside of the ROI. We define the estimated parameters of $u$ and $v$ as $\hat{u}$ and $\hat{v}$ respectively. The axial component of the displacement, $\hat{v}$, is the motion used to compute the strain map. By estimating $\hat{v}$ for all the windows inside the ROI we obtain a map of axial displacements denoted as $d(x, y)$.

Once the axial displacements are estimated in the ROI, the values of the strain are computed using the strain filter proposed by Kallel and Ophir [17]. They show that a filter expressed as (7) convolved with the axial component of the motion estimation $d(x, y)$ can generate a smooth strain map $\varepsilon(x, y)$.

$$
g(n)=\xi(n)\left[\begin{array}{ll}
1 & -\frac{n+1}{2}
\end{array}\right]\left[\begin{array}{llll}
1 & 2 & \ldots & n \\
1 & 1 & \ldots & 1
\end{array}\right]
$$

where $\xi(n)=\frac{12}{n\left(n^{2}-1\right)}$ and $n$ is the number of samples in the interval $\Delta_{y}$.

The strain map $\varepsilon(x, y)$ provides the elastic information inside the ROI. This map is changing constantly due to lateral motion of the probe and the non-constant compression motion. In order to improve the quality of the strain map also 
known as elastogram, we use its information as the input of the visual servoing approach presented in the next section.

\section{B. Control law}

In this section, we consider a convex ultrasound probe attached to the end-effector of a 6-DOF robotic arm. The different Cartesian reference frames involved are depicted in Fig. 3. The frame $\mathcal{F}_{s}$ is attached to a 6-axes ATI Gamma 65-SI force/torque sensor, the frame $\mathcal{F}_{e}$ to the end-effector of the robot, the frame $\mathcal{F}_{g}$ to the gravity center of mass of the ultrasound probe and $\mathcal{F}_{p c}$ is the frame attached to the contact point between the probe and the object surface. The frames $\mathcal{F}_{p}$ and $\mathcal{F}_{r}$ are, respectively, the image frame positioned at the center of the convex probe and the center of the ROI, indicated by the user, where the elastography process is performed.

The objective of the proposed robotic task is to perform the automatic palpation motion while moving the probe to obtain several strain maps at different $\theta_{z}$ angles in the image plane of the probe. Registering and averaging together the different estimated strain maps, which are usually very noisy, will then improve the quality of the elastogram. This is achieved by controlling the ultrasound probe in-plane velocity (3 DOF) at the point of contact frame $\mathcal{F}_{p c}, \mathbf{v}=\left[\begin{array}{lll}v_{x} & v_{y} & \omega_{z}\end{array}\right]^{\top}$. The robotic-assisted elastography involves three simultaneous tasks: i) force control to continuously generate slight tissues deformation, ii) visual control to horizontally position the element of interest (bounded by the ROI) in the center of the whole image in order to keep it visible, and iii) orientation control of the ultrasound probe to obtain different view angles of the ROI for image quality improvement after registration and averaging processes between the estimated strain maps.

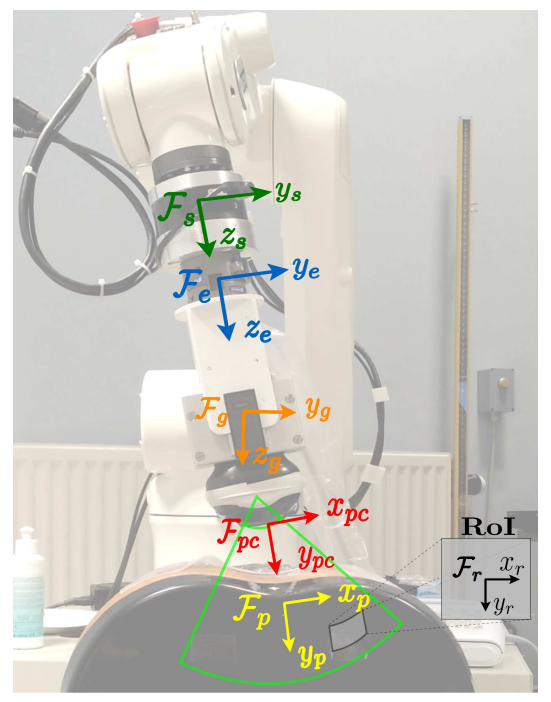

Fig. 3. Cartesian reference frames attached to the robotic arm.

1) Force control: We apply a force control law to perform the compression motion between the probe and the object being scanned to follow a desired sinusoidal force variation value. We recall briefly the force control scheme as described in [10]. The robot is equipped with a 6-axes force/torque sensor providing a force tensor measurement ${ }^{s} \mathbf{H}_{s}$ expressed in the sensor frame $\mathcal{F}_{s}$. The gravity force tensor is ${ }^{g} \mathbf{H}_{g}=\left[\begin{array}{lllllll}0 & 0 & 9.81 m_{p} & 0 & 0 & 0\end{array}\right]^{\top}$ expressed in the gravity frame $\mathcal{F}_{g}$, where $m_{p}$ is the mass of the probe. The force transformation matrices ${ }^{s} \mathbf{F}_{g}$ and ${ }^{p c} \mathbf{F}_{s}$ are derived from the known relative positions between the frames. ${ }^{s} \mathbf{F}_{g}{ }^{g} \mathbf{H}_{g}$ is the gravity force tensor expressed in the sensor frame $\mathcal{F}_{s}$, and ${ }^{p c} \mathbf{F}_{s}$ allows expressing the force tensor in the point contact frame $\mathcal{F}_{p c}$. Therefore, the force tensor ${ }^{p c} \mathbf{H}_{p c}$ expressed in the frame $\mathcal{F}_{p c}$ can be computed by:

$$
{ }^{p c} \mathbf{H}_{p c}={ }^{p c} \mathbf{F}_{s}\left({ }^{s} \mathbf{H}_{s}-{ }^{s} \mathbf{F}_{g}{ }^{g} \mathbf{H}_{g}\right)
$$

As we want to control the force only along the $y$-axis of $\mathcal{F}_{p}$, we define the (scalar) force control feature as $\mathbf{s}_{f}=$ $\left[\begin{array}{llllll}0 & 1 & 0 & 0 & 0 & 0\end{array}{ }^{p c} \mathbf{H}_{p c}\right.$. The change of the force feature through time can be expressed as:

$$
\dot{\mathbf{s}}_{f}=\mathbf{L}_{f} \mathbf{v}
$$

where $\mathbf{L}_{f}=\left[\begin{array}{lll}0 & k & 0\end{array}\right]$ is an interaction matrix that relates the force feature to the velocity of the probe $\mathbf{v}$, with $k$ being an estimation of the contact stiffness. We define the force error $\mathbf{e}_{f}$ to minimize as the difference between the force feature $\mathbf{s}_{f}$ and a desired force $\mathbf{s}_{f}^{*}, \mathbf{e}_{f}=\mathbf{s}_{f}-\mathbf{s}_{f}^{*}$. To reach an exponential decrease of this force error, we define the desire force error variation as $\dot{\mathbf{e}}_{f}^{*}=-\lambda_{f} \mathbf{e}_{f}$ with $\lambda_{f}$ being the force control gain. To perform the motion of compression between the probe and the object being scanned, we propose a sinusoidal change of $\mathbf{s}_{f}^{*}$ through time using a function as shown in Fig. 4, defined as:

$$
\mathbf{s}_{f}^{*}(\tau)=F(\tau)=\frac{\Delta_{F}}{2}\left[\sin \left(\frac{(4 \tau-T) \pi}{2 T}\right)+1\right]+F_{0}
$$

where $\tau \geq 0$ is the discrete sample iteration number (sampled at control frequency $f_{s c}$ ), $T$ the period of the curve expressed in number of sample time, $F_{0}$ the initial force, and $\Delta_{F}$ the amplitude of the sinusoidal force signal we add to obtain a repetitive compression of the soft tissue.

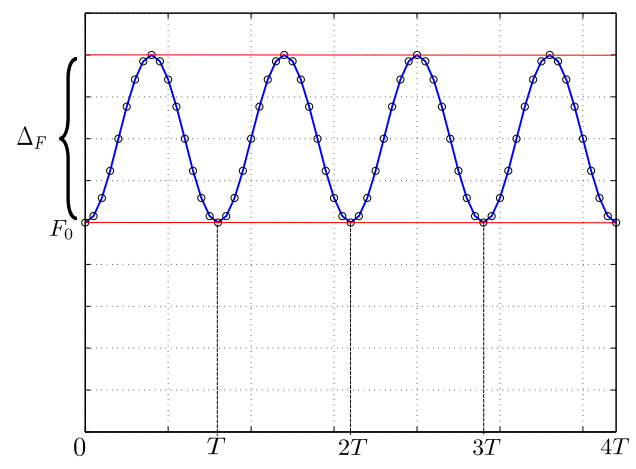

Fig. 4. Desired sinusoidal function proposed for the force control.

2) Horizontal centering of the target: We use the elastogram to automatically center a stiff object at the middle of the full image. First, we propose to generate an image 
$I_{g}(x, y)$ by filtering the strain map with a gaussian function as:

$$
I_{g}(x, y)=\frac{e^{-\varepsilon(x, y)^{2}}}{e^{\varepsilon_{\max }^{2}}}
$$

where $\varepsilon_{\max }=\max (|\varepsilon(x, y)|)$ and $I_{g}(x, y) \in[0,1]$. The aim of this function is to enhance the intensity of the rigid objects and to decrease the intensity of the rest of the area. Then, we compute its center of gravity, $\left(x_{c}, y_{c}\right)$ using the 2D image moments as proposed in [18]. To horizontally center the rigid object in the full image, we minimize $x_{c}$ to zero. From a basic kinematic relation we obtain the interaction matrix $\mathbf{L}_{t}=\left[\begin{array}{lll}-1 & 0 & y_{c}\end{array}\right]$ that links the variation of the visual feature $s_{t}$ to the probe velocity:

$$
\dot{\mathbf{s}}_{t}=\mathbf{L}_{t} \mathbf{v}
$$

The visual error is defined as $\mathbf{e}_{t}=\mathbf{s}_{t}-\mathbf{s}_{t}^{*}$, where $\mathbf{s}_{t}=x_{c}$ and $\mathbf{s}_{t}^{*}$ is the desired visual feature that we fix to zero (center of the image). We impose the desire visual error variation to be $\dot{\mathbf{e}}_{t}^{*}=-\lambda_{t} \mathbf{e}_{t}$ with $\lambda_{t}$ being the visual control gain.

3) Probe orientation: The aim of this task is to automatically orient the probe to a desired angle $\mathbf{s}_{\theta}^{*}$ (in the image plane) from the current angle of the probe $\mathbf{s}_{\theta}=\theta-\theta_{\text {init }}$. $\theta_{\text {init }}$ is the angle of the initial probe orientation and $\theta$ is the angle measured during the probe orientation control. Both angles are obtained by the odometry measures of the robot. The variation of the angle feature $\mathbf{s}_{\theta}$ due to the probe velocity is defined as:

$$
\dot{\mathbf{s}}_{\theta}=\mathbf{L}_{\theta} \mathbf{v}
$$

where $\mathbf{L}_{\theta}=\left[\begin{array}{lll}0 & 0 & -1\end{array}\right]$ is the interaction matrix related to $\mathbf{s}_{\theta}$. The angle error is defined as $\mathbf{e}_{\theta}=\mathbf{s}_{\theta}-\mathbf{s}_{\theta}^{*}$, and we impose the desired angle error variation to be $\dot{\mathbf{e}}_{\theta}^{*}=-\lambda_{\theta} \mathbf{e}_{\theta}$ with $\lambda_{\theta}$ being the probe orientation control gain.

4) General control law: We define $\mathbf{L}$ as the general interaction matrix composed by $\mathbf{L}_{f}, \mathbf{L}_{t}$ and $\mathbf{L}_{\theta}$ :

$$
\mathbf{L}=\left[\begin{array}{l}
\mathbf{L}_{t} \\
\mathbf{L}_{f} \\
\mathbf{L}_{\theta}
\end{array}\right]=\left[\begin{array}{ccc}
-1 & 0 & y_{c} \\
0 & k & 0 \\
0 & 0 & -1
\end{array}\right]
$$

and the control law is defined as:

$$
\mathbf{v}=\mathbf{L}^{-1}\left[\begin{array}{c}
-\lambda_{t} \mathbf{e}_{t} \\
-\lambda_{f} \mathbf{e}_{f} \\
-\lambda_{\theta} \mathbf{e}_{\theta}
\end{array}\right]
$$

It is important to remark that the matrix $\mathbf{L}$ is directly invertible. Therefore, the general control law can be reduced as:

$$
\mathbf{v}=\left[\begin{array}{c}
\lambda_{t} \mathbf{e}_{t}+y_{c} \lambda_{\theta} \mathbf{e}_{\theta} \\
-\frac{\lambda_{f} \mathbf{e}_{f}}{k} \\
\lambda_{\theta} \mathbf{e}_{\theta}
\end{array}\right]
$$

\section{RESUlTS}

We present the results in two sections. In the first part, we validate our elastography real-time process on synthetic and experimental data. In the second part, we present experimental results using our control approach to improve the elastogram quality in a ROI indicated by the user.

\section{A. Elastography validation with synthetic data}

The pre-compress RF signals were simulated from FIELD II [19]. Based on the technical features of the Ultrasonix probe C5-2/60, a 128-element linear array transducer with a center frequency of $3.5 \mathrm{MHz}$ was modeled. The transducer has a pitch of $468 \mu \mathrm{m}$ and an element height of $13 \mathrm{~mm}$. Each RF array includes 100 RF scan lines. The probe covers a depth of $80 \mathrm{~mm}$ and a width of $50 \mathrm{~mm}$. Random scatterers were distributed with random amplitudes within a 2D homogeneous model with dimensions $50 \times 60 \mathrm{~mm}$. The Young's modulus of the model was $25 \mathrm{kPa}$. A stiff object with cylindrical shape of $10 \mathrm{~mm}$-diameter and an Young's modulus of $75 \mathrm{kPa}$ was embedded in the center of the model. The Poisson's ratio of the model was 0.495 , and the model contains $10^{5}$ scatterers. The scatterers were then moved according to solutions from finite element analysis at different applied forces $\Delta_{F}$. The simulations with finite element analysis were performed using the software COMSOL 5.0 and MATLAB 8.0. The post-compress RF signals were then simulated from Field II using the new scatterer distribution (see Fig. 5).

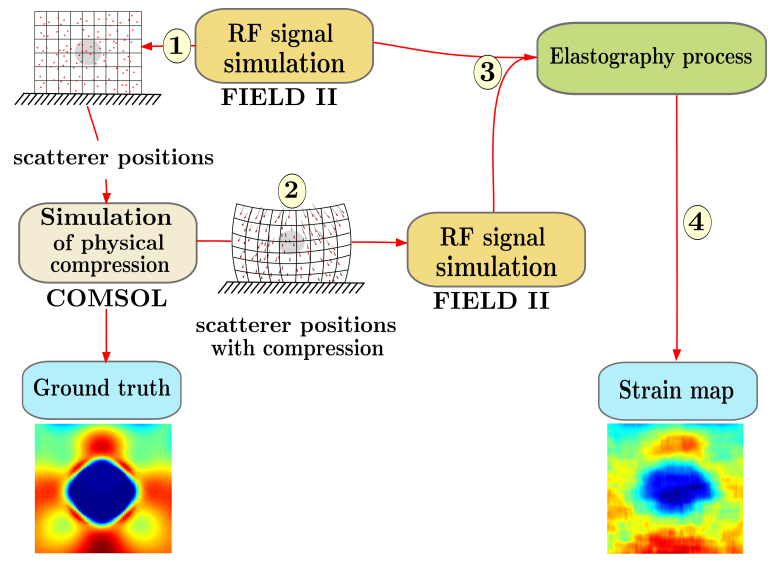

Fig. 5. Elastography simulation.

The elastography process described in II-A was coded in $\mathrm{C}++$, and takes as input the pre- and post-compress $\mathrm{RF}$ signals (see Fig. 5). The elastography output is the estimated elastogram of the pre- and post-compress RF signals. The obtained process time of the elastography algorithm was 20 ms corresponding to $50 \mathrm{fps}$ (frames/second), over a ROI of $50 \%$ of the RF frame size. Therefore, it is compatible with the time constraint of a robotic control scheme. The minimum force variation $\Delta_{F}=2 N$ required was found by applying different forces in the finite element analysis. The lower relative error compared with the ground truth computed by the finite element analysis was $5.3 \%$, which proves that our elastogram is well estimated.

\section{B. Experimental results using a training abdominal phantom}

For all experiments we used a Viper s850 robot (Adept Technology Inc., USA) and an Ultrasonix 4DC7-3/40 convex ultrasound probe (used in 2D imaging mode) rigidly attached to its end-effector. The ultrasound frames were grabbed 
from the SonixTouch ultrasound system and processed on a workstation (Intel Xeon CPU @2.1 GHz), which computes and sends the control law to the robot. The RF frames were captured at a frequency of $23 \mathrm{~Hz}$. The force control law was performed with a higher frequency $(200 \mathrm{~Hz})$. The visual control was performed with the same period as the image capture (23 fps). The experiments were performed over an ABDFAN ultrasound examination training model (Kyoto Kagaku Co., Japan) simulating the abdomen of a patient. The phantom manufacturer specifications notes the presence of lesions and tumors. Experiments were performed selecting a ROI including hepatic lesions and pancreatic tumors.

In the experiments, the probe was initially positioned above the phantom, without contact, and oriented with an initial angle $\theta_{\text {init }}$ and initial force $F_{0}=0$. Then, to demonstrate the efficiency of the general control law (16) we set a desired sinusoidal force signal with $F_{0}=5 \mathrm{~N}$ and $\Delta_{F}=2 \mathrm{~N}$. The automatic horizontal centering of the ROI is activated once the user selects this area in a graphical interface developed for this work. We performed several experiments using the set-up previous described. We present the details for one of the experiments. A set of five desired angles for the probe orientation is used: $\theta_{0}=\theta_{\text {init }}-10^{\circ}$, $\theta_{1}=\theta_{0}+5^{\circ}, \theta_{2}=\theta_{1}+5^{\circ}, \theta_{3}=\theta_{2}+5^{\circ}$ and $\theta_{4}=\theta_{3}+5^{\circ}$. The curves of error for the three tasks in this experiment are shown in Fig. 6. We can see that the force error ranges between $\pm 1 \mathrm{~N}$ due to the sinusoidal desired force variation. Once the ROI is selected (at time $20 \mathrm{~s}$ ), the object centering error also decreases towards zero but still exhibits a low remaining oscillation of $\pm 3 \mathrm{~mm}$ due to the elastography noise. However, the ROI is horizontally maintained close to the image center even when the user successively changed the probe desired angles at times 21, 90, 126, 167 and 205 $\mathrm{s}$, keeping automatically the object of interest in the field of view. Fig. 7 presents in the second row the overlay of the b-mode US images and the elastograms, showing that the elasticity map (ROI) has reached the horizontal center of the full image for each probe orientation.

To improve the elastography quality, we propose to align and average the different elastograms obtained for all probe orientations. This alignment is performed by a warping function that consists in applying a translation based on the centroid relative position and the image relative rotation between each elastogram of the object of interest (blue region in the images of the third row in the Fig. 7, where dark blue is the lower strain, and dark red is the highest strain). Once we obtain the warped elastograms and average them together, the result will ensure an improvement of the elastography quality compared with only one elastogram obtained for a given probe orientation. The result of this quality improvement is shown in Fig. 8.

Further, in elastography, the concept of contrast-noiseratio $\left(\mathrm{CNR}_{e}\right)$ allows to make a decision on accepting or rejecting the presence of a lesion as presented in [20], and a higher level of $\mathrm{CNR}_{e}$ suggests better ability to detect the lesion. Therefore, to evaluate the elastography quality in

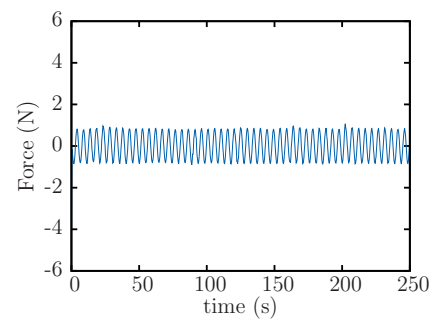

(a) Force error measured

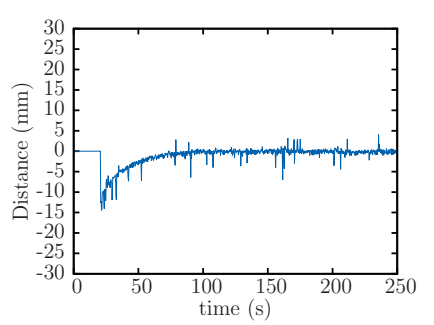

(c) Horizontal target centering error

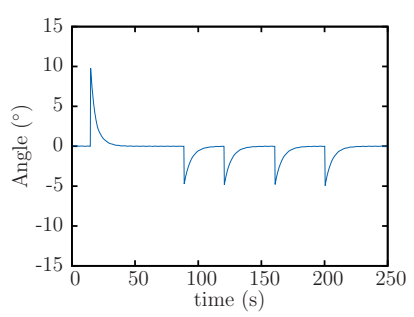

(b) Orientation error curve

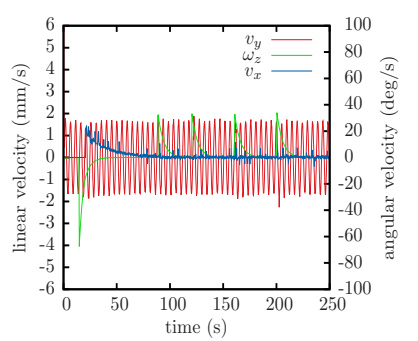

(d) Velocities
Fig. 6. Phantom experiment. Evolution of the system during the experiment. (a) Force error curve, with $F_{0}=5 \mathrm{~N}$ and $\Delta_{F}=2 \mathrm{~N}$. (b) Probe orientation error curve. (c) Horizontal target centering error curve. (d) Velocities of the 3-DOFs involved in the control law.

\begin{tabular}{|c|c|c|c|c|c|}
\hline$\theta_{0}$ & $\theta_{1}$ & $\theta_{2}$ & $\theta_{3}$ & $\theta_{4}$ & mean \\
\hline 97.40 & 71.45 & 65.87 & 87.52 & 87.02 & 127.42 \\
\hline
\end{tabular}

TABLE I. Comparison of the $\mathrm{CNR}_{e}$ in $\mathrm{dB}$ of estimated elastography images at the different probe orientations of the experiment and their mean.

our experiment, we compute the $\mathrm{CNR}_{e}$ in the elastography images for each probe orientation and for their mean as shown in Table I. The highest $\mathrm{CNR}_{e}$ is obtained for the image of the mean of the elastograms as we expected.

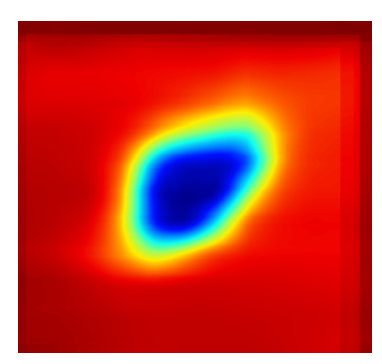

Fig. 8. Mean of aligned elastograms obtained from 5 probe orientations.

\section{CONCLUSION}

We proposed a new approach for automating the palpation motion required for quantitative elastography that allows also its quality improvement. The control is based on three tasks that respectively generate the soft tissue deformation required for ultrasound elastography, keeping the region of interest centered in the image and changing the probe orientation to observe the object of interest with different angles of view. The average of the warped elasticity maps computed from different probe orientations shows a better 


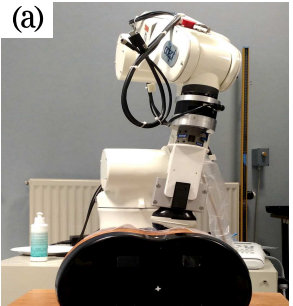

(f)

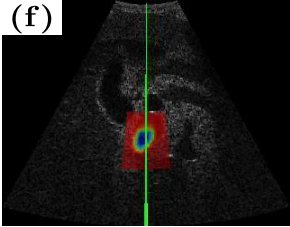

(k)

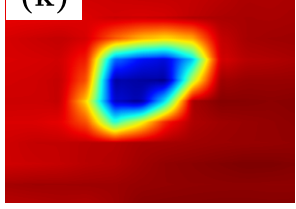

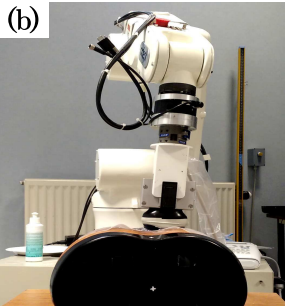

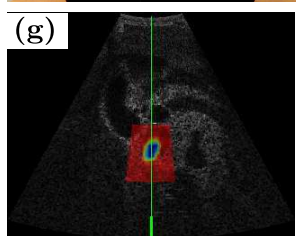

(1)

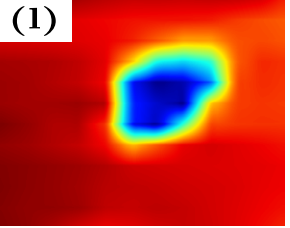

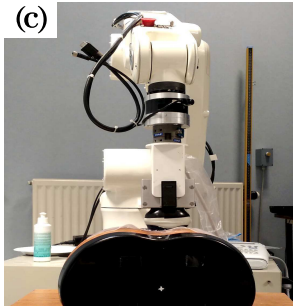
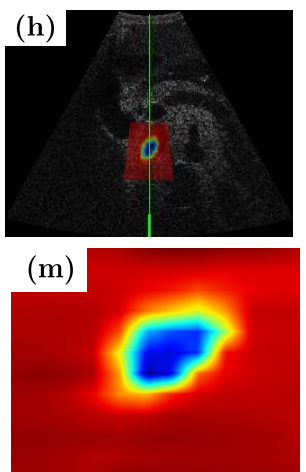
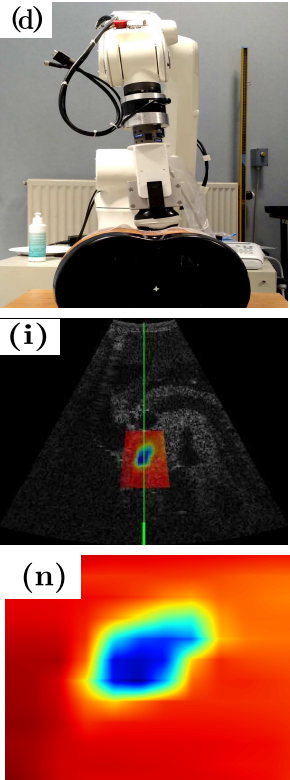
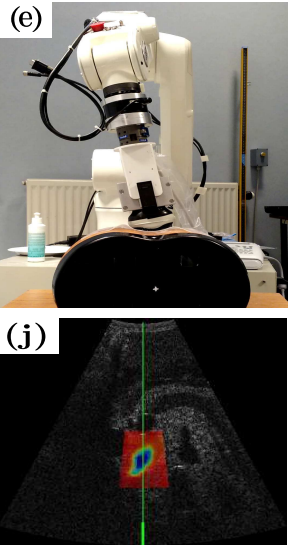

(o)

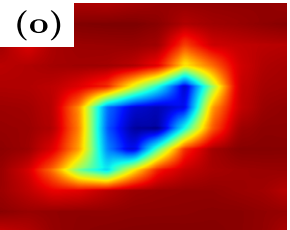

Fig. 7. Experiment with different probe orientations: (a)-(e) show the pictures with the probe oriented at different angles, from left to right the angles are $\theta_{0}=-10^{\circ}, \theta_{1}=-5^{\circ}, \theta_{2}=0^{\circ}, \theta_{3}=5^{\circ}$ and $\theta_{4}=10^{\circ}$. (f)-(j) show the b-mode image when the target is centered with the image and the ROI overlaid for each probe orientation. The elastography obtained for each probe orientation is shown in the ROI of the images (k)-(o).

quality elastogram compared with the ones obtained from a unique probe orientation. In future work we will extend this approach to the use of a 3D ultrasound probe to provide volumetric elastography information of soft tissue.

\section{ACKNOWLEDGMENT}

This work has been jointly supported by Inria Rennes Bretagne Atlantique and the National Council of Science and Technology of Mexico (CONACYT) under the Grant no. $242120 / 383765$.

\section{REFERENCES}

[1] M. Pompili, L. Riccardi, M. Covino, B. Barbaro, C. Di Stasi, R. Orefice, G. Gasbarrini, and G. L. Rapaccini, "Contrast-enhanced gray-scale harmonic ultrasound in the efficacy assessment of ablation treatments for hepatocellular carcinoma," Liver International, vol. 25, pp. 954-961, Oct. 2005.

[2] L. Solbiati, T. Ierace, M. Tonolini, and L. Cova, "Guidance and monitoring of radiofrequency liver tumor ablation with contrast-enhanced ultrasound," European Journal of Radiology, vol. 51, Supplement, pp. S19-S23, Feb. 2004.

[3] E. Turgay, S. Salcudean, and R. Rohling, "Identifying the mechanical properties of tissue by ultrasound strain imaging," Ultrasound in Medicine \& Biology, vol. 32, no. 2, pp. 221-235, 2006.

[4] Z. Wen-Jing, W. Qing, Y. Yi-Yi, F. Qian-Jin, and C. Wu-Fan, "Ultrasound elasticity imaging of thyroid using analytic minimization," in Control Conference (CCC), 2012 31st Chinese, July 2012, pp. 40184020.

[5] H. Lin, X. Chen, Y. Guo, Y. Shen, and S. Chen, "Quantitative shear elasticity assessment of liver fibrosis in rat model with shear wave elastography base on acoustic radiation force," in Medical Biometrics, 2014 International Conference on, May 2014, pp. 131-134.

[6] S. Billings, N. Deshmukh, H. J. Kang, R. Taylor, and E. M. Boctor, "System for robot-assisted real-time laparoscopic ultrasound elastography," vol. 8316, 2012, pp. $83161 \mathrm{~W}-83161 \mathrm{~W}-8$.

[7] C. Schneider, A. Baghani, R. Rohling, and S. Salcudean, "Remote ultrasound palpation for robotic interventions using absolute elastography," 2012, pp. 42-49.
[8] M. A. Bell, Sen, I. Iordachita, and P. Kazanzides, "Force-controlled ultrasound robot for consistent tissue pre-loading: Implications for acoustic radiation force elasticity imaging," in Biomedical Robotics and Biomechatronics (2014 5th IEEE RAS EMBS International Conference on, Aug. 2014, pp. 259-264.

[9] K. Nichols and A. Okamura, "Methods to segment hard inclusions in soft tissue during autonomous robotic palpation," Robotics, IEEE Transactions on, vol. 31, no. 2, pp. 344-354, April 2015.

[10] P. Chatelain, A. Krupa, and N. Navab, "Optimization of ultrasound image quality via visual servoing," in Robotics and Automation (ICRA), 2015 IEEE International Conference on, May 2015, pp. 59976002.

[11] M. Rao, Q. Chen, H. Shi, and T. Varghese, "Spatial-angular compounding for elastography using beam steering on linear array transducers," Med Phys, vol. 33, no. 3, pp. 618-626, Mar 2006.

[12] J. Ophir, "Elastography: A quantitative method for imaging the elasticity of biological tissues," Ultrasonic Imaging, vol. 13, no. 2, pp. 111-134, Apr. 1991.

[13] A. Pesavento, C. Perrey, M. Krueger, and H. Ermert, "A time-efficient and accurate strain estimation concept for ultrasonic elastography using iterative phase zero estimation," Ultrasonics, Ferroelectrics, and Frequency Control, IEEE Transactions on, vol. 46, no. 5, pp. 10571067, Sept. 1999.

[14] T. Loupas, J. Powers, and R. Gill, "An axial velocity estimator for ultrasound blood flow imaging, based on a full evaluation of the doppler equation by means of a two-dimensional autocorrelation approach," Ultrasonics, Ferroelectrics, and Frequency Control, IEEE Transactions on, vol. 42, no. 4, pp. 672-688, July 1995.

[15] X. Pan, J. Gao, S. Tao, K. Liu, J. Bai, and J. Luo, "A two-step optical flow method for strain estimation in elastography: Simulation and phantom study," Ultrasonics, vol. 54, no. 4, pp. 990 - 996, 2014.

[16] B. K. P. Horn, Ed., Robot Vision. Cambridge, MA, USA: MIT Press, 1986.

[17] F. Kallel and J. Ophir, "A least-squares strain estimator for elastography," Ultrason Imaging, vol. 19, no. 3, pp. 195-208, Jul 1997.

[18] F. Chaumette, "Image moments: a general and useful set of features for visual servoing," Robotics, IEEE Transactions on, vol. 20, no. 4, pp. 713-723, Aug 2004.

[19] J. A. Jensen and P. Munk, "Computer phantoms for simulating ultrasound b-mode and cfm images," in Acoustical imaging, 1997, pp. 75-80.

[20] T. Varghese and J. Ophir, "An analysis of elastographic contrast-tonoise ratio," Ultrasound in Medicine \& Biology, vol. 24, no. 6, pp. $915-924,1998$. 